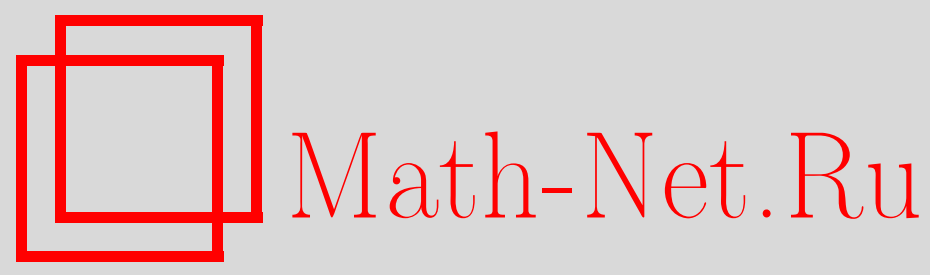

С. А. Шкарин, Об эффективных линейно упорядоченных множествах функций сравнения, Матем. заметки, 2000, том 67, выпуск 4, 629-637

DOI: https://doi.org/10.4213/mzm878

Использование Общероссийского математического портала Math-Net.Ru подразумевает, что вы прочитали и согласны с пользовательским соглашением http://www.mathnet.ru/rus/agreement

Параметры загрузки:

IP : 54.205 .225 .156

26 апреля 2023 г., 16:58:51

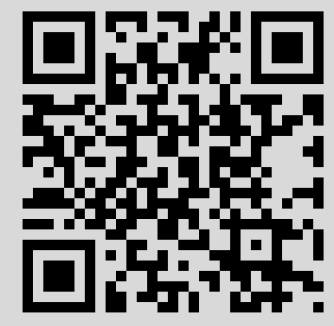




\title{
ОБ ЭФФЕКТИВНЫХ ЛИНЕЙНО УПОРЯДОЧЕННЫХ МНОЖЕСТВАХ ФУНКЦИЙ СРАВНЕНИЯ
}

\section{C. А. Шкарин}

\begin{abstract}
C аксиоматикой ZFC теории множеств совместимы как наличие, так и отсутствие линейно упорядоченного (некоторыми естественньми отношениями порядка) эфффективного множества функций сравнения (=плотного класса сравнения).

Библиограффия: 10 названий.
\end{abstract}

1. Введение. Напомним несколько определений. Функиией сравнения называется целая функция $A(z)=\sum_{n=0}^{\infty} A_{n} z^{n}$ такая, что $A_{n}>0$ при всех ${ }^{1} n \in \omega$ и последовательность $\alpha_{n}=A_{n+1} / A_{n}$ убывает (возможно, нестрого) и стремится к нулю. Всюду ниже для целой функции $A$ (соответственно $B$ или $C$, быть может, с верхними индексами) символами $A_{n}\left(B_{n}, C_{n}\right)$ будем обозначать коэффициенты Тейлора функции $A(B, C)$, а символами $\alpha_{n}\left(\beta_{n}, \gamma_{n}\right)$ - величины $A_{n+1} / A_{n}\left(B_{n+1} / B_{n}, C_{n+1} / C_{n}\right)$. Мы будем придерживаться следуюших обозначений: $\mathscr{H}$ - множество всех целых функций, $\mathscr{P} \subset \mathscr{H}$ - множество всех полиномов, $\mathscr{H}^{\circ}=\mathscr{H} \backslash \mathscr{P}, \mathscr{A}$ - множество всех функций сравнения. Типом функиии $F \in \mathscr{H}$ относительно $A \in \mathscr{A}$ назовем число

$$
\sigma_{A}(F)=\inf \{\tau \in(0, \infty):|F(z)|<A(\tau|z|) \text { при }|z|>R=R(\tau)\} .
$$

Известная теорема об $A$-типе, утверждает, что

$$
\sigma_{A}(F)=\varlimsup_{n \rightarrow \infty}\left(\frac{\left|F_{n}\right|}{A_{n}}\right)^{1 / n}
$$

Функции сравнения играют значительную роль в теории интерполяции аналитических функций [1]- [3], [4]. Информация о функциях сравнения имеется в [5]. Несколько другая, но сходная характеристика роста целых функций предлагается в [4]. Приведенное определение является естественным обобщением понятия экспоненциального типа целой функции: экспоненциальный тип - это тип относительно $A(z)=e^{z}$. Число $\sigma_{A}(F)$ дает содержательную информацию о росте функции $F$, если $0<\sigma_{A}(F)<\infty$. Это обстоятельство оправдывает введение понятия эффективного множества функций сравнения. Множество $M \subset \mathscr{A}$ называется әффективныц (или плотным классом сравнения), если для всякой функции $F \in \mathscr{H}^{\circ}$ существует $A \in M$ такая, что $0<\sigma_{A}(F)<\infty$.

\footnotetext{
${ }^{1}$ Здесь и ниже $\omega=\{0,1,2, \ldots\}$ - множество целых неотрицательных чисел.
} 
В этой работе рассматривается вопрос, поставленный Ю. А. Казьминьг, о существовании эффективного множества функций сравнения, линейно упорядоченного относительно естественно возникающих частичных упорядочений множества $\mathscr{A}$. Пусть $A, B \in \mathscr{A}$. Тогда соотношения порядка определяются так:

1) $A<_{0} B \Longleftrightarrow \lim _{n \rightarrow \infty}\left(A_{n} / B_{n}\right)^{1 / n}=0$;

2) $A<_{1} B \Longleftrightarrow \lim _{n \rightarrow \infty}\left(\alpha_{n} / \beta_{n}\right)=0$;

3) $A<_{2} B \Longleftrightarrow \lim _{n \rightarrow \infty}\left(\alpha_{n} / \beta_{n}\right)=0$ и последовательность $\alpha_{n} / \beta_{n}$ финально убывает. $^{2}$

Легко видеть, что $A<_{2} B \Longrightarrow A<_{1} B \Longrightarrow A<_{0} B$ и что условие $A<_{0} B$ эквивалентно условию $\sigma_{B}(A)=0$.

Напомним, что множеством первой категории в топологическом пространстве назьвается объединение счетного числа ниг де не плотных множеств; прочие множества назьваются множествами второй категории.

Пусть СН - континуум-гипотеза, a LC - следующее условие.

УСловиЕ LC. Отрезок нельзя представить в виде объединения линейно упорядоченного (по включению) набора множеств первой категории.

Еще в 30-х годах Гёдель доказал (см., например, [6]), что СН совместима с аксиоматикой теории множеств ZFC (см. [6], [7]) Цермело-Френкеля (с аксиомой выбора). Из основного результата работы [8] вытекает следующая теорема. Эта теорема совпадает также с утверждением 12 из [9] для случая $m=\aleph_{2}$.

ТЕорема VH. С аксиоматикой ZFC совместимо следующее сочетание свойств:

1) $2^{\aleph_{0}}=\aleph_{2}$;

2) отрезок $[0,1]$ не является обвединением $\aleph_{1}$ множеств 1-й категории;

3) на отрезке $[0,1]$ существует мнохсество второй категории мощности $\aleph_{1}$.

\section{СлЕДСТВИЕ 1. Условие LC совместимо с ZFC.}

ДоКАЗАТЕЛЬСТво. Нам достаточно проверить, что условие LC вьполняется в модели из теоремы VH. Предположим, что это не так. Тогда $d[0,1]=\bigcup_{\alpha<\tau} X_{\alpha}$, где $\tau-$ некоторый кардинал, $\alpha$ пробегает множество ординалов, меньших $\tau, X_{\alpha}$ - множества первой категории в $[0,1]$ и $X_{\alpha} \subset X_{\beta}$ при $\alpha<\beta$. По свойству 2 из теоремы VH $\tau>\aleph_{1}$. Следовательно, $\tau=\aleph_{2}$, ибо $|[0,1]|=\aleph_{2}$ по свойству 1 . По свойству 3 существует множество второй категории $Y \subset[0,1]$ такое, что $|Y|=\aleph_{1}$. В силу последнего равенства существует $\alpha<\tau$, для которого $Y \subset X_{\alpha}$. Получилось противоречие с тем, что $X_{\alpha}-$ множество первой категории. Следствие доказано.

В настоящей работе доказываются следующие две теоремы.

Теорема 1. В предположении справедливости СН существует әффективное линейно упорядоченное отношением $<2$ подмнохество $\mathscr{B} \subset \mathscr{A}$.

ТЕОРема 2. В предполохсении справедливости LC для всякого линейно упорядоченного отношением $<_{0}$ множества $\mathscr{B} \subset \mathscr{A}$ существует функиия $f \in \mathscr{H}$

\footnotetext{
${ }^{2}$ Последовательность $a_{n}$ вещественных чисел назьвается финально убывающей, если $a_{n+1} \leqslant$ $a_{n}$ при всех достаточно больших $n$.
} 
такая, что $\sigma_{B}(f)=\infty$ для всех $B \in \mathscr{B}$. Тем самым, не существует линейно упорядоченного отношением $<_{0}$ әффективного множества функций сравнения.

Из теорем 1, 2 и следствия 1 вытекает

СлеДСТВИЕ 2. Для любого $i \in\{0,1,2\}$ утверждение о существовании линейно упорядоченного отношением $<_{i}$ эффективного подмножества $\mathscr{B} \subset \mathscr{A}$ не зависит от ZFC, т.е. это утверэсдение и его отрицание - оба совместимы с ZFC.

2. Доказательство теоремы 1. Для доказательства нам потребуется ряд технических лемм.

Лемма 1. Пусть $A^{[n]}, B^{[n]} \in \mathscr{A}(n \in \omega), F \in \mathscr{H}$, причем $A^{[n]}<2 B^{[m]}, A^{[n]}<2$ $A^{[n+1]}, B^{[n+1]}<_{2} B^{[n]}, \sigma_{A^{[n]}}(F)=\infty, \sigma_{B^{[n]}}(F)=0$ npu всеx $m, n \in \omega$. Tогдa существует $B \in \mathscr{A}$ такая, что $\sigma_{B}(F)=0 u A^{[n]}<_{2} B<_{2} B^{[n]}$ nрu всех $n \in \omega$.

ДокАЗАТЕльство. Пусть $n_{k}$ - строговозрастающая последовательность натуральных чисел. Положим $\beta_{k}=\beta_{k}^{[1]}$ при $k \leqslant n_{1} ; \beta_{k} / \beta_{k-1}=\beta_{k}^{[m]} / \beta_{k-1}^{[m]}$ при $n_{m-1}<k \leqslant n_{m}$, $m \geqslant 2$. Очевидно, приведенные соотношения однозначно определяют последовательность $\beta_{n}$. Положим также $B_{0}=1, B_{n}=\prod_{k=0}^{n-1} \beta_{k}$ при $n>0$ и (пока чисто формально) $B(z)=\sum_{k=0}^{\infty} B_{k} z^{k}$. Для доказательства леммы достаточно показать, что при подходящем выборе $n_{k}$ вьшолняются условия:

(1.1) последовательность $\beta_{k}$ монотонно убывает и стремится к нулю (следовательно, $B_{n}-$ коэффициенты Тейлора функции сравнения $\left.B\right)$;

(1.2) $\sigma_{B}(F)=0$ и $A^{[n]}<_{2} B<_{2} B^{[n]}$ для всех $n \in \omega$.

Опишем процесс выбора последовательности $n_{k}$. Отметим, что в силу (1.1) и определения порядка $<_{2}$ все достаточно большие натуральные числа $k$ удовлетворяют условиям

$$
\frac{\beta_{k+1}^{[2]}}{\beta_{k+1}^{[1]}} \leqslant \frac{\beta_{k}^{[2]}}{\beta_{k}^{[1]}} \quad \text { и } \frac{\alpha_{k+1}^{[1]}}{\beta_{k+1}^{[2]}} \leqslant \frac{\alpha_{k}^{[1]}}{\beta_{k}^{[2]}}
$$

(1.b) $F_{k} \leqslant \prod_{j=0}^{k-1} \beta_{j}^{(2)}$, где $\beta_{j}^{(2)}$ определяется из соотношений $\beta_{k}^{(2)}=\beta_{k}^{[1]}$ при $k \leqslant n_{1}$; $\beta_{k}^{(2)} / \beta_{k-1}^{(2)}=\beta_{k}^{[2]} / \beta_{k-1}^{[2]}$ при $n_{1}<k$.

Натуральное число $n_{1}$ надо выбрать так, чтобы при всех $k \geqslant n_{1}$ вьполнялись условия (1.a) и (1.b). Далее, если $n_{1}, \ldots, n_{j-1}$ уже выбраны, то $n_{j} \in \omega$ выберем так, чтобы $n_{j}>n_{j-1}$ и при всех $k \geqslant n_{j}, 1 \leqslant i \leqslant j$ выполнялись условия

$$
\frac{\beta_{k+1}^{[j+1]}}{\beta_{k+1}^{[i]}} \leqslant \frac{\beta_{k}^{[j+1]}}{\beta_{k}^{[i]}} \text { и } \frac{\alpha_{k+1}^{[i]}}{\beta_{k+1}^{[j+1]}} \leqslant \frac{\alpha_{k}^{[i]}}{\beta_{k}^{[j+1]}} ;
$$

$\left(1 . \mathrm{b}^{\prime}\right) F_{k} \leqslant j^{-k} \prod_{p=0}^{k-1} \beta_{p}^{(j+1)}$, где $\beta_{p}^{(j+1)}$ определяется из соотношений:

$$
\begin{array}{ll}
\beta_{p}^{(j+1)}=\beta_{p}^{[1]} & \text { при } p \leqslant n_{1} ; \\
\frac{\beta_{p}^{(j+1)}}{\beta_{p-1}^{(j+1)}}=\frac{\beta_{p}^{[m]}}{\beta_{p-1}^{[m]}} \quad \text { при } n_{m-1}<p \leqslant n_{m}, \quad 2 \leqslant m \leqslant j ; \\
\frac{\beta_{p}^{(j+1)}}{\beta_{p-1}^{(j+1)}}=\frac{\beta_{p}^{[j+1]}}{\beta_{p-1}^{[j+1]}} \quad \text { при } p>n_{j} .
\end{array}
$$


Отметим ряд свойств $\beta_{n}$, соответствующих построенной последовательности $n_{k}$.

1) Из построения $n_{k}$ непосредственно видно, что $\beta_{k} / \beta_{k-1} \leqslant 1$ при всех $k$. Значит, последовательность $\beta_{n}$ убывает.

2) Из условия (1.a') видно, что

$$
\frac{\beta_{k}}{\beta_{k-1}}=\frac{\beta_{k}^{[m]}}{\beta_{k-1}^{[m]}} \leqslant \frac{\beta_{k}^{[i]}}{\beta_{k-1}^{[i]}} \quad \text { при } n_{m-1}<k \leqslant n_{m}, \quad i \leqslant m \text {. }
$$

Следовательно, $\beta_{k} / \beta_{k}^{[i]} \leqslant \beta_{k-1} / \beta_{k-1}^{[i]}$. Таким образом, для всякого $i$ последовательность $\beta_{k} / \beta_{k}^{[i]}$ финально убьвает. Отсюда и из убьвания $B^{[i]}$ по порядку $<2$ получаем, что $\lim _{k \rightarrow \infty}\left(\beta_{k} / \beta_{k}^{[i]}\right)=0$. Из последнего равенства и 1$)$ видно, что $B \in \mathscr{A}_{\text {и }} B<_{2} B^{[i]}$ при всех $i$.

3) Из условия (1.a') вытекает, что

$$
\frac{\beta_{k}}{\beta_{k-1}}=\frac{\beta_{k}^{[m]}}{\beta_{k-1}^{[m]}} \geqslant \frac{\alpha_{k}^{[i]}}{\alpha_{k-1}^{[i]}} \quad \text { при } n_{m-1}<k \leqslant n_{m}, \quad i \leqslant m \text {. }
$$

Стало быть, последовательность $\alpha_{k}^{[i]} / \beta_{k}$ финально убывает.

4) Из условия $\left(1 . \mathrm{b}^{\prime}\right)$ следует, что $\left(F_{k} / B_{k}\right)^{1 / k} \leqslant \frac{1}{m}$ при $n_{m-1}<k \leqslant n_{m}$. Значит, $\sigma_{B}(F)=0$.

$5)$ Из 3) и равенств $\sigma_{A^{[n]}}(F)=\infty, \sigma_{B^{[n]}}(F)=0$ имеем, что $\lim _{k \rightarrow \infty}\left(\alpha_{k}^{[i]} / \beta_{k}\right)=0$ для всякого $i \in \omega$. Стало быть, $A^{[i]}<_{2} B$. Лемма доказана.

Лемма 2. Пусть $A, B^{[n]} \in \mathscr{A}(n \in \omega), F \in \mathscr{H}$, причем $\sigma_{A}(F)=\infty u A<_{2} B^{[n]}$,

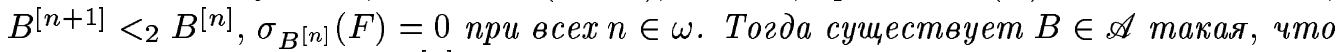
$\sigma_{B}(F)=0$ u $A<_{2} B<_{2} B^{[n]} \partial \Omega_{\text {я }}$ всеx $n \in \omega$.

ДокАЗАТЕЛЬСТво аналогично доказательству леммы 1 (но несколько проще).

Лемма 3. Пусть $B \in \mathscr{A}, A^{[n]}(n \in \omega), F \in \mathscr{H}$, причем $\sigma_{B}(F)=0 u A^{[n]}<_{2} B$, $A^{[n]}<_{2} A^{[n+1]}, \sigma_{A^{[n]}}(F)=\infty$ nри всех $n \in \omega$. Тогда существует $A \in \mathscr{A}$ такая, что $\sigma_{A}(F)=\infty u A^{[n]}<_{2} A<_{2} B$ для всех $n \in \omega$.

ДокАЗАТЕЛьСтво. Будем действовать так же, как при доказательстве леммы 1. Пусть $n_{k}$ - строго возрастающая последовательность натуральных чисел. Положим $\alpha_{k}=\alpha_{k}^{[1]}$ при $k \leqslant n_{1} ; \alpha_{k} / \alpha_{k-1}=\alpha_{k}^{[m]} / \alpha_{k-1}^{[m]}$ при $n_{m-1}<k \leqslant n_{m}, m \geqslant 2$. Функцию $A$ определим по $\alpha_{n}$ так же, как функция $B$ определялась по $\beta_{n}$ в доказательстве леммы 1. Нам остается выбрать подходящим образом последовательность $n_{k}$. Так же как при доказательстве леммы $1, n_{1}$ выберем так, чтобы вьполнялись условия:

$$
\frac{\alpha_{k+1}^{[1]}}{\alpha_{k+1}^{[2]}} \leqslant \frac{\alpha_{k}^{[1]}}{\alpha_{k}^{[2]}} \quad \text { и } \quad \frac{\alpha_{k+1}^{[2]}}{\beta_{k+1}} \leqslant \frac{\alpha_{k}^{[2]}}{\beta_{k}} \text { для всех } k \geqslant n_{1} .
$$

(3.b) Существует $m<n_{1}$ такое, что $F_{m} \geqslant \prod_{j=0}^{m-1} \alpha_{j}$, где $\alpha_{j}=\alpha_{j}^{[1]}$ при $j \leqslant n_{1}$. 
Далее, если $n_{1}, \ldots, n_{j-1}$ уже выбраны, то $n_{j} \in \omega$ выберем так, чтобы $n_{j}>n_{j-1}$ и при всех $k \geqslant n_{j}, 1 \leqslant i \leqslant j$ выполнялись условия:

$$
\frac{\alpha_{k+1}^{[i]}}{\alpha_{k+1}^{[j+1]}} \leqslant \frac{\alpha_{k}^{[i]}}{\alpha_{k}^{[j+1]}} \quad \text { и } \quad \frac{\alpha_{k+1}^{[j+1]}}{\beta_{k+1}} \leqslant \frac{\alpha_{k}^{[j+1]}}{\beta_{k}} .
$$

$\left(3 . \mathrm{b}^{\prime}\right)$ Существует $m \in\left(n_{j-1}, n_{j}\right)$ такое, что $F_{m} \geqslant j^{m} \prod_{p=0}^{m-1} \alpha_{p}$, где $\alpha_{p}$ определяется из соотношений: $\alpha_{p}=\alpha_{p}^{[1]}$ при $p \leqslant n_{1} ; \alpha_{p} / \alpha_{p-1}=\alpha_{p}^{[r]} / \alpha_{p-1}^{[r]}$ при $n_{r-1}<p \leqslant n_{r}$, $2 \leqslant r \leqslant j$.

Так же как при доказательстве леммы 1 , из свойств $\left(3 . \mathrm{a}^{\prime}\right)$ и $\left(3 . \mathrm{b}^{\prime}\right)$ вьводятся следуюшие свойства последовательности $\alpha_{n}$ (и соответствующих $A_{n}, A(z)$ ).

1) Последовательность $\alpha_{n}$ убывает.

2) Из условия (3.a') видно, что

$$
\frac{\alpha_{k}^{[i]}}{\alpha_{k-1}^{[i]}} \leqslant \frac{\alpha_{k}}{\alpha_{k-1}}=\frac{\alpha_{k}^{[m]}}{\alpha_{k-1}^{[m]}} \leqslant \frac{\beta_{k}}{\beta_{k-1}} \quad \text { при } \quad n_{m-1}<k \leqslant n_{m}, \quad i \leqslant m .
$$

Значит, последовательности $\alpha_{k} / \beta_{k}, \alpha_{k} / \alpha_{k}^{[i]}$ финально убьвают.

3) Из 2) и 1) следует, что $A$ - функция сравнения. В силу произвольности $i$, возрастания последовательности $A^{[i]}$ по порядку $<_{2}$ и 2 ) получаем, что $A^{[i]}<_{2} A$ при всех $i$.

4) Из $\left(3 . \mathrm{b}^{\prime}\right)$ следует, что $\sigma_{A}(F)=\infty$. Из последнего равенства и 2) вытекает, что $\lim _{k \rightarrow \infty}\left(\alpha_{k} / \beta_{k}\right)=0$. Значит, $A<_{2} B$. Лемма доказана.

Лемма 4. Пусть $A, B \in \mathscr{A}, F \in \mathscr{H}$, причем $A<_{2} B, \sigma_{A}(F)=\infty u \sigma_{B}(F)=0$. Тогда существует $C \in \mathscr{A}$ такая, что $A<_{2} C<_{2} B$ и $\sigma_{C}(F)=1$.

ДоказАТЕЛЬСТво. Так как $\lim _{n \rightarrow \infty}\left(\left|F_{n}\right| / B_{n}\right)^{1 / n}=0$ и $F \neq 0$, то

$$
0<r=\max _{n \in \omega}\left(\frac{\left|F_{n}\right|}{B_{n}}\right)^{1 / n}<\infty
$$

В силу определения отношения $<_{2}$ существует $n_{1} \in \omega$ такое, что $\alpha_{n+1} / \beta_{n+1} \leqslant \alpha_{n} / \beta_{n}$ при $n \geqslant n_{1}$. Положим $\gamma_{i}=r \beta_{i}$ при $i \leqslant n_{1}$ и $\gamma_{n}$ при $n>n_{1}$ будем выбирать индуктивно. Опишем шаг индукции. Пусть $\gamma_{0}, \gamma_{1}, \ldots, \gamma_{N}\left(N \geqslant n_{1}\right)$ уже выбраны, причем

$$
\frac{\gamma_{n+1}}{\gamma_{n}} \in\left[\frac{\alpha_{n+1}}{\alpha_{n}}, \frac{\beta_{n+1}}{\beta_{n}}\right] \quad \text { при } n_{1} \leqslant n \leqslant N-1
$$

(непустота последнего отрезка очевидна в силу выбора $n_{1}$ );

$$
\begin{gathered}
\text { если } \gamma_{j}^{\prime}= \begin{cases}\gamma_{j} & \text { при } 0 \leqslant j \leqslant N, \\
\gamma_{N} \beta_{j} / \beta_{N} & \text { при } j>N,\end{cases} \\
C_{0}^{\prime}=1, \quad C_{j}^{\prime}=\prod_{k=0}^{j-1} \gamma_{k}^{\prime} \quad \text { при } j \geqslant 1, \quad \text { то } \Delta_{N}=\max _{k \in \omega}\left(\frac{\left|F_{k}\right|}{C_{k}^{\prime}}\right)^{1 / k} \leqslant 1 .
\end{gathered}
$$


Пусть $\Delta_{N}^{\prime}=\max _{k} \geqslant N+1\left(\left|F_{k}\right| / C_{k}^{\prime}\right)^{1 / k} \leqslant 1$. Очевидно, $\Delta_{N}^{\prime} \leqslant \Delta_{N}$ Выберем $\gamma_{N+1}$ и проследим за выполнением условий (4.1) и (4.2). Для $\delta \in\left[\gamma_{N} \alpha_{N+1} / \alpha_{N}, \gamma_{N} \beta_{N+1} / \beta_{N}\right]$ положим

$$
\gamma_{j}^{N, \delta}= \begin{cases}\gamma_{j} & \text { при } 0 \leqslant j \leqslant N \\ \delta \frac{\beta_{j}}{\beta_{N+1}} & \text { при } j>N .\end{cases}
$$

Отметим, что из выбора $\delta$ следует, что $\gamma_{j}^{N, \delta} \leqslant \gamma_{j}^{\prime}$ и $\Delta_{N}^{\gamma_{j}^{\prime}}=\Delta_{N}^{\prime} \leqslant \Delta_{N} \leqslant 1$, где

$$
\Delta_{N}^{\delta}=\max _{k \geqslant N+1}\left(\frac{\left|F_{k}\right|}{C_{k}^{N, \delta}}\right)^{1 / k}, \quad C_{j}^{N, \delta}=\prod_{k=0}^{j-1} \gamma_{k}^{N, \delta}
$$

Из определения величины $\Delta_{N}^{\delta}$ следует, что функция $\delta \mapsto \Delta_{N}^{\delta}$ строго убывает и непрерывна. Последнее утверждение делает корректным следующее определение величины $\gamma_{N+1}$. Пусть $\gamma_{N+1}=\gamma_{N} \alpha_{N+1} / \alpha_{N}$, если $\Delta_{N}^{\delta}<1$ при всех $\delta \in\left[\gamma_{N} \alpha_{N+1} / \alpha_{N}, \gamma_{N} \times\right.$ $\left.\beta_{N+1} / \beta_{N}\right]$. В противном случае в качестве $\gamma_{N+1}$ возьмем (единственное) число $\delta \in\left[\gamma_{N} \times\right.$ $\left.\alpha_{N+1} / \alpha_{N}, \gamma_{N} \beta_{N+1} / \beta_{N}\right]$ такое, что $\Delta_{N}^{\delta}=1$. Вьполнение условий (4.1) и (4.2) для $\gamma_{0}$, $\gamma_{1}, \ldots, \gamma_{N+1}$ непосредственно вытекает из построения $\gamma_{N+1}$. Описание выбора последовательности $\gamma_{n}$ закончено. Положим $C_{n}=\prod_{k=0}^{n-1} \gamma_{k}$. Из условия (4.2) имеем, что

$$
\left(\frac{\left|F_{k}\right|}{C_{k}}\right)^{1 / k} \leqslant 1 \quad \forall k \in \omega .
$$

Из условия (4.1) следует, что последовательности $\gamma_{n} / \beta_{n}, \alpha_{n} / \gamma_{n}$ финально убьвают (точнее - убывают при $n \geqslant n_{1}$ ). Учитьвая, что последовательность $\beta_{n}$ убьвает и стремится к нулю ( $B$ - функция сравнения), получаем, что последовательность $\gamma_{n}$ обладает теми же свойствами и, следовательно, $C(z)=\sum_{n=0}^{\infty} C_{n} z^{n}-$ функция сравнения. Из $(1)$ видно, что $\sigma_{C}(F) \leqslant 1$. Заметим, что равенство $\Delta_{N}^{\gamma_{N+1}}=1$ выполняется для бесконечного числа значений $N$ (иначе, при $N$, больших некоторого $N_{0}$, вьполняется равенство $\gamma_{N+1}=\gamma_{N} \alpha_{N+1} / \alpha_{N}$, следовательно, $\gamma_{N}=\mu \alpha_{N}$ для некоторой положительной константы $\mu$ и $\varlimsup_{n \rightarrow \infty}\left(\left|F_{n}\right| / C_{n}\right)^{1 / n}=\infty$ в силу условия $\sigma_{A}(F)=\infty$, что противоречит (1)). Условие $\Delta_{N}^{\gamma_{N+1}}=1$ эквивалентно существованию $m>N$ такого, что $C_{m}=F_{m}$. Таким образом, равенство $C_{m}=F_{m}$ выполняется для бесконечного числа значений $m$. Стало быть, $\sigma_{C}(F) \geqslant 1$. То есть, $\sigma_{C}(F)=1$. Из финального убывания последовательностей $\gamma_{n} / \beta_{n}, \alpha_{n} / \gamma_{n}$ следует, что обе они имеют (конечньй) предел. Но если бы первьй из этих двух пределов был бы отличен от нуля, то вьшло бы противоречие с условием $\sigma_{B}(F)=0$, а если бы отличен от нуля был второй предел, то это противоречило бы условию $\sigma_{A}(F)=\infty$ (здесь используется равенство $\sigma_{C}(F)=1$ ). Равенство нулю этих пределов влечет соотношение $A<_{2} C<_{2} B$. Лемма доказана.

Следующие два утверждения проверяются элементарно.

Лемма 5. Пусть $M \subset \mathscr{A}-$ (не более чем) счетное множество (возможно пустое), $F \in \mathscr{H}, \sigma_{A}(F)=\infty$ для всех $A \in M$. Тогда существует $B \in \mathscr{A}$ такая, что $\sigma_{B}(F)=0$ u $A<_{2} B$ для всех $A \in M$.

Лемма 6. Пусть $M \subset \mathscr{A}$ - счетное множество, $F \in \mathscr{H}^{\circ}, \sigma_{B}(F)=0$ при всех $B \in M$. Тогда существует $A \in \mathscr{A}$ такая, что $\sigma_{A}(F)=\infty u A<_{2} B$ при всех $B \in M$. 
ОСНовНАЯ ЛЕмма. Пусть $M \subset \mathscr{A}$-счетное множество, линейно упорядоченное отношением $<_{2}, F \in \mathscr{H}^{\circ}$. Тогда существует $M^{\prime} \subset \mathscr{A}$ такое, что $M \subseteq M^{\prime}$ $u$

(O.1) $M^{\prime}$ - счетное мноэсество, линейно упорядоченное отношением $<_{2}$;

(O.2) Cуществует $C \in M^{\prime}$ такое, что $0<\sigma_{C}(F)<\infty$.

ДоказАтЕльство. Если существует $C \in M$ такое, что $0<\sigma_{C}(F)<\infty$, то само множество $M$ подойдет в качестве $M^{\prime}$. Если такой функции $C$ не существует, то $M=M_{1} \cup M_{2}$, где $M_{1}=\left\{A \in M: \sigma_{A}(F)=\infty\right\}, M_{2}=\left\{A \in M: \sigma_{A}(F)=0\right\}$. B силу линейной упорядоченности множества $M$ отношением $<_{2}$ множества $M_{1}$ и $M_{2}$ также линейно упорядочены этим отношением, причем $A<_{2} B$ при всех $A \in M_{1}, B \in M_{2}$. Возможны несколько случаев.

СлучАй 1. Множество $M_{1}$ непусто и не имеет наибольшего элемента (относительно порядка $\left.<_{2}\right)$, а множество $M_{2}$ непусто и не имеет наименьшего элемента.

В силу счетности линейно упорядоченных множеств $M_{1}$ и $M_{2}$ существуют последовательности $A^{[n]} \in M_{1}, B^{[n]} \in M_{2}$ такие, что $A^{[n]}<_{2} A^{[n+1]}$ и $B^{[n+1]}<_{2} B^{[n]}$ для всех $n \in \omega$, и для любых $G \in M_{1}, H \in M_{2}$ существует $n \in \omega$ такое, что $G<_{2} A^{[n]}$ и $B^{[n]}<_{2} H$.

Теперь нетрудно заметить, что функции $A^{[n]}, B^{[n]}$ и $F$ удовлетворяют условиям леммы 1. Пусть $B$ - функция, существование которой утверждается в лемме 1 . Теперь функции $A^{[n]}, B$ и $F$ удовлетворяют условиям леммы 3 и пусть $A$ - функция, существование которой гарантируется леммой 3 . Функции $A, B$ и $F$, очевидно, удовлетворяют условиям леммы 4. Теперь видно, что в качестве $M^{\prime}$ можно взять множество $M \cup\{C\}$, где $C$ - функция из леммы 4 . В самом деле, из перечисленных лемм и свойств последовательностей $A^{[n]}$ и $B^{[n]}$ следует, что $\sigma_{C}(F)=1$ и $A<_{2} C<_{2} B$ для всех $A \in M_{1}$, $B \in M_{2}$.

СлучАй 2. Множество $M_{1}$ непусто и не имеет наибольшего элемента, а множество $M_{2}$ имеет наименьший элемент $B$.

Выберем последовательность $A^{[n]} \in M_{1}$ такую, что $A^{[n]}<{ }_{2} A^{[n+1]}$ для всех $n \in \omega$ и для любой $G \in M_{1}$ существует $n \in \omega$ такое, что $G<{ }_{2} A^{[n]}$.

Тогда функции $A^{[n]}, B, F$ удовлетворяют условиям леммы 3 . Пусть $A$ - функция, сушествование которой доказано в лемме 3 . Функции $A, B$ и $F$, очевидно, удовлетворяют условиям леммы 4 . Из лемм 3,4 , так же как в первом случае, следует, что в качестве $M^{\prime}$ можно взять множество $M \cup\{C\}$, где $C$ - функция из леммы 4.

СлучАй 3. Множество $M_{1}$ имеет наибольший элемент $A$, а множество $M_{2}$ непусто и не имеет наименьшего элемента.

Выберем последовательность $B^{[n]} \in M_{2}$ такую, что $B^{[n+1]}<{ }_{2} B^{[n]}$ для всех $n \in \omega$ и для любой $H \in M_{2}$ существует $n \in \omega$ такое, что $B^{[n]}<_{2} H$.

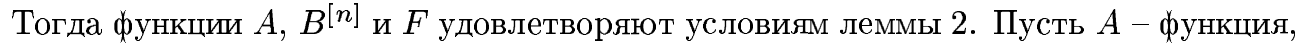
существование которой доказано в лемме 2. Существование искомого множества получается применением леммы 4 так же, как в предыдущем случае.

СлучАЙ 4. Множество $M_{1}$ имеет наибольший элемент $A$, а множество $M_{2}$ имеет наименьший элемент $B$.

Доказательство существования множества $M^{\prime}$ совпадает с завершающим этапом доказательства в трех разобранных случаях (т.е. мгновенно следует из леммы 4). 
СлучАЙ 5. $M_{1}=\varnothing$ или $M_{2}=\varnothing$.

В этом случае леммы 5 и 6 позволяют добавить к множеству $M$ один элемент (или два элемента, если оба множества $M_{1}$ и $M_{2}$ пусты) так, чтобы множество $M$ по-прежнему оставалось линейно упорядоченным, а множества $M_{1}$ и $M_{2}$ стали бы непустыми. В этом случае мы окажемся в условиях одного из предыдущих случаев. Лемма доказана.

ДоКАЗАТЕЛЬСТво ТЕОРЕМЫ 1. Пусть вьполняется СН. Тогда $\mathscr{H}^{\circ}=\left\{F_{\alpha}: \alpha<\aleph_{1}\right\}$ ( $\alpha$ пробегает множество счетных ординалов). Построим индуктивно трансфинитную последовательность $\left\{M_{\alpha}: \alpha<\aleph_{1}\right\}$ счетных подмножеств $\mathscr{A}$, обладающих следующими свойствами:

1. $M_{\alpha} \subset M_{\beta}$ при $\alpha<\beta$;

2. $M_{\alpha}$ линейно упорядочено отношением $<_{2}$;

3. Для всякого $\alpha<\beta$ найдется $A \in M_{\beta}$ такое, что $0<\sigma_{A}\left(F_{\alpha}\right)<\infty$.

Положим $M_{0}=\varnothing$. Пусть $\beta<\aleph_{1}$ и $M_{\alpha}$ при $\alpha<\beta$, удовлетворяющие требуемьп условиям, построены. Построим $M_{\beta}$. Если $\beta$ - предельньй ординал, то в качестве $M_{\beta}$ можно взять $\bigcup_{\alpha<\beta} M_{\alpha}$ (это множество счетно в силу счетности ординала $\beta$ ). Если $\beta$ имеет непосредственного предшественника $\beta-1$, то в качестве $M_{\beta}$ можно взять множество $M^{\prime}$, которое получается, если применить основную лемму к $F=F_{\beta-1}, M=M_{\beta-1}$. Построение $M_{\alpha}$ завершено. Из условий $1,2,3$, которым удовлетворяют множества $M_{\alpha}$, непосредственно следует, что $\mathscr{B}=\bigcup_{\alpha<\aleph_{1}} M_{\alpha}$ - эффективное линейно упорядоченное отношением $<2$ множество функций сравнения.

ЗАмЕЧАнИЕ 1 . Если $\mathscr{B}$ - эффективное линейно упорядоченное отношением $<_{0}$ множество функций сравнения, то для всякой функции $F \in \mathscr{H}^{\circ}$ существует ровно одна функция $B \in \mathscr{B}$ такая, что $0<\sigma_{B}(F)<\infty$.

ЗАмЕчАниЕ 2. Схема доказательства теоремы 2 позволяет (в предположении СН) всякое линейно упорядоченное (порядком <2) сепарабельное (в порядковой топологии) множество функций сравнения дополнить до эффективного линейно упорядоченного множества.

ДоКАЗАТЕЛЬСТво тЕоРЕмы 2. Символом $c_{0}$ будем обозначать банахово пространство всех комплексных последовательностей, сходящихся к нулю (с нормой $\|x\|=$ $\left.\max \left\{\left|x_{n}\right|: n \in \omega\right\}\right)$.

ЛЕмма 7. В предположсении справедливости LC пространство со нельзя представить в виде оббединения линейно упорядоченного (по включению) множества подмножсеств первой категории.

ДокАЗАтЕльство. Очевидно, достаточно показать существования биективного отображения $f:[0,1] \rightarrow c_{0}$ такого, что отображения $f$ и $f^{-1}$ переводят любое множество первой категории в множество первой категории. Существование такого отображения вытекает из теоремы 2 с. 495 в книге [10]. Лемма доказана.

Перейдем непосредственно к доказательству теоремы 2 . Пусть $\mathscr{B}-$ линейно упорядоченное отношением $<_{0}$ множество функций сравнения. Для всякого $B \in \mathscr{B}$ положим

$$
Z_{B}=\left\{x \in c_{0}: \varlimsup_{n \rightarrow \infty} \frac{\left|x_{n}\right|}{B_{n}^{1 / n}}<\infty\right\} .
$$


Из определения порядка $<_{0}$ следует, что $Z_{B} \subset Z_{C}$ при $B<_{0} C$. Значит, множество $\left\{Z_{B}: B \in \mathscr{B}\right\}$ - линейно упорядоченное по включению множество, состоящее из подмножеств $c_{0}$. Предположим, что заключение теоремы 2 не выполняется. Тогда всякая целая функция имеет конечньй тип относительно некоторой функции из $\mathscr{B}$. Следовательно, $\bigcup_{B \in \mathscr{B}} Z_{B}=c_{0}$. Стало быть, по лемме 7 найдется $B \in \mathscr{B}$ такое, что $Z_{B}-$ множество второй категории. Но $Z_{B}=\bigcup_{m \in \omega} L_{m}$, где $L_{m}=\left\{x \in c_{0}:\left|x_{n}\right| \leqslant m B_{n}^{1 / n}\right.$ для всех $n \in \omega\}$. Следовательно, среди множеств $L_{m}$ есть множества второй категории. С другой стороны каждое $L_{m}-$ компактное подмножество $c_{0}$ и, значит, нигде не плотно (ибо всякое компактное подмножество бесконечномерного банахового пространства ниг де не плотно). Полученное противоречие доказьвает теорему 2.

ЗАмЕчаниЕ 3. Автору неизвестно, совместимо ли с ZFC утверждение о наличии эффективного линейно упорядоченного отношением $<_{i}$ множества функций сравнения и об одновременном отсутствии такого множества, упорядоченного отношением $<j$ при некоторых $i, j \in\{0,1,2\}, i<j$.

\section{СПИСОК ЦИТИРОВАННОЙ ЛИТЕРАТУРЫ}

[1] Казьмин Ю.А. Сравнения функция // Математическая энциклопедия. Т. 5: Советская энциклопедия, 1985. С. 168.

[2] Казьмин А. Ю. Об одной задаче А. О. Гельфонда // Матем. сб. 1973. Т. 90. № 4. С. 521-543.

[3] Казьмин А. Ю. Об интерполировании средними // Докл. АН СССР. 1987. Т. 295. № 5. C. $1050-1053$.

[4] Осколков А. В. О некоторых вопросах теории целых функций // Матем. сб. 1993. Т. 184. № 1. C. $129-148$.

[5] Казьмин Ю.А. Методы теории интерполяции аналитических функций и их приложения. Дисс.... д. ф.-м.н. М.: МГУ, 1972.

[6] Дж. Барвайс (ред. ) Справочная книга по математической логике. Т. 2. Теория множеств. М.: Мир, 1980.

[7] Куратовский К., Мостовский А. Теория множеств. М.: Мир, 1970.

[8] Vopenka P., Hrbachek K. The consistency of some theorem of real numbers // Bull. Acad. Polon. Sci., ser. sci. math., astr. et phis. 1967. V. 15. № 3. P. 107-111.

[9] Малыхин В. И. Топология и форсинг // УМН. 1983. Т. 38. № 1. С. 69-118.

[10] Куратовский К. Топология. Т. 1. М.: Мир, 1966. 